\title{
Leveraging a Model towards Efficient Energy Management: A Survey
}

\author{
Nikita Parab \\ Computer Engineering \\ VESIT, University of Mumbai \\ Mumbai, Maharashtra
}

\author{
Himanshu Pandita \\ Computer Engineering \\ VESIT, University of Mumbai \\ Mumbai, Maharashtra
}

\author{
Karan Patel \\ Computer Engineering \\ VESIT, University of Mumbai \\ Mumbai, Maharashtra
}

\author{
Prashant Brahme \\ Computer Engineering \\ VESIT, University of Mumbai \\ Mumbai, Maharashtra
}

\author{
Gresha Bhatia \\ Computer Department \\ VESIT, University of Mumbai \\ Mumbai,Maharashtra
}

\begin{abstract}
This paper focuses on the shortcomings of the existing power management system and proposes an alternate methodology to achieve quick and efficient power restoration. Various restoration algorithms and technologies such as Q-learning, clustering algorithm and decision trees are discussed in this paper. Two way communication mechanism (ICT) is implemented with the help of Smart Grids which manages the ever changing demands of energy by continuously monitoring and controlling the grid's transmission system. Consumers get an opportunity to actively participate in restoration plans with the help of ICT. The proposed system will help to identify, isolate and restore the faults in the shortest time possible with the help of ICT.
\end{abstract}

\section{Keywords}

Smart Grids, Agents, Self-healing grid, islanding, Multi Agent System (MAS)

\section{INTRODUCTION}

To supply electricity from the producer to the consumers , electrical grids are used. The power is generated in the power plant from where the power is stepped up using a step up transformer to increase the voltage before transmission so as to reduce power loss over long distances. This stepped up power is then transferred using high voltage transmission line to the substation step down transformer which brings voltage to a level which is suitable for low voltage circuits. The stepped down power is then transferred using low voltage transmission lines to different consumers [1].

The traditional electrical grid has many limitations such as:

- Inefficiency at managing peak load: The peak load describes a period where demand of power is more than the average supply of power. So to comply with the excess demand, a buffer of excess supply is maintained, which results in less efficiency and high costs. So, as the demand for power increases, the traditional system becomes more unstable.

- $\quad$ Limited Delivery System: The existing grid system suffers from a limited bandwidth and slow data transmission rates of electricity which severely affects the response time. Also there is no transparency in the distributed network below substation. As a result, the time taken for power restoration also increases due to limited bandwidth.

- Cannot integrate renewable: The traditional grid is not capable to transmit power generated from renewable resources. This would cause more strain on the already depleting traditional resources.

- Almost zero customer participation: This system only allows one way communication, i.e. only the utility company has the information about the consumers and their power consumption. Whereas the customer is not involved in any power related activities. Less participation results in lack of transparency between the system and the customer.

- Slow power restoration: Whenever a fault occurs, the utility company sends a field crew member to the fault location who isolates the faults, identifies the fault and tries to restore load to as many impacted customer as possible. This process took time and the during the power outage many industries suffered heavy losses [2].

For the past decade, the telecommunication companies have been looking for ways to reduce electricity bills, their consumption and emissions and reduce the power restoration time. To find an optimal solution for this, vast amount of research has been conducted in fields of wired, wireless and optical communication. The result of this research was introduction of smart grids which can further brings down the bills, reduce energy consumption and emission by the telecommunication companies and uses an automatic mechanism for fault detection, isolation and restoration [3].

A smart grid is an electricity network based on digital technology that is used to supply electricity to consumers via two-way communication. This paper mainly focuses on the power restoration in smart grids. Now a days in some grids more than $80 \%$ power outages results from faults in distribution networks. Restoration is an essential function required by distribution utilities to secure reliable service and reduce outage time. The smart grid hosts a variety of powerful technologies that help enable automatic service restoration, more efficient crew dispatch and, most importantly, faster service restoration. 


\section{SMART GRID}

\subsection{What is Smart Grid?}

Smart Grid is a system that continuously senses the transmission lines and provides a two way communication between the utilities and the consumers. It uses technologies such as Phasor Measurement Units (PMU) which work with the existing electric grids and respond quickly to the ever changing energy demands by continuously monitoring and controlling the grid's transmission system. During power outages, Smart Grid technologies helps to detect and isolate power lines in order to prevent large-scale blackouts. These technologies will also ensure quick and strategic recovery of electricity. It helps to create awareness among consumers about the connection between the electricity use and the environment with the help of "Smart meters" and other mechanisms.

The various features of Smart Grids are:

- Reliability: Technologies such as state estimation are used by the smart grids to improve fault detection and perform self-healing of the network.

- Flexibility: The transmission and distribution infrastructure of SG allows bidirectional energy flows which results in distributed generation from photovoltaic panels on building roofs, fuel cells, batteries of electric cars, wind turbines and hydroelectric power.

- Efficiency: The deployment of smart grid technologies such as the demand side management results in the overall improvement of the efficiency of energy infrastructure. For example, reducing voltage on distribution lines through Voltage/VAR Optimization (VVO), reducing truck rolls using data from Advanced Metering Infrastructure systems.

- Demand Response support: Generators and loads interact and coordinate in real time in an automated fashion in real time using Demand Response support.

\subsection{Smart meters and home energy management systems}

Smart meters operate digitally and act as a Smart Grid interface between the user and the energy provider. It will transfer automated and complex information from the energy provider which will help the user to cut down on the energy costs. An Energy Management System (EMS) will help the user to track the energy usage in detail. It will also help the user to monitor real time information and signals from the utilities and create automatic setting for optimum power usage. It can turn off specific appliances and equipment automatically when a large demand threatens a power outage, thus preventing blackouts by avoiding peak demand rates and balancing the energy load in the area. In case of a power outage, the Smart Grid, with its advanced technologies, will allow a consumer to utilize power from "distributed renewable resources," such as solar power, hydro power, and wind energy, until the workers can restore the power. This process is known as "islanding".

\section{Renewable Energy}

The fastest growing sources of renewable power such as the wind and solar power cannot be put to use efficiently using the current power grid. Integrating these renewable sources of energy in the current grid is very difficult. Smart grids will provide grid operators new tools and energy storage capabilities to absorb excess wind and solar energy. This energy storage will help to cope up with the uncertainties in wind and solar resources thus making it easier to use them.

\subsection{Consumer Engagement}

The Smart Grid provides various opportunities for consumers to conserve energy and for utilities to make use of the grid efficiently. By participating in such programs, the consumers can earn extra savings on their money bills. Some mechanisms such as time of use pricing, net metering and compensation programs for plugin electric vehicles (PEVs) are used to save energy on a large extent

Time of use programs: The demand for energy changes throughout the day depending on the time of the day and the weather conditions. When the demand is highest, the workload of power plants and utilities is more which may often lead to blackouts and reduction in the voltage of the system. Time of use programs encourages consumers to use more power when the demand is less by reducing the rates of power consumption. A steady and reliable stream of electricity is made available to everyone by evenly distributing the demand of power.

Net metering: This mechanism is used by the users which generate their own power at home using rooftop solar power and so on. An advanced meter is used which calculates the power transferred back to the grid as credit. Using the Smart Grid technology, power utilities can pay more for consumer generated power during peak power demand and less for off peak power. Such a pricing structure and financial incentives will encourage active participation of consumers in efficient power management system.

\subsection{Distribution}

A vital feature of distribution intelligence is fault detection and response. Distribution intelligence helps to identify the source of a power outage so that field crew can be notified about the fault through communication. An automated system overcomes the shortcomings of the traditional system by responding to power outages more quickly and keep the power flowing to customers. By combining sensors and automated switching, an intelligent system can determine the best approach to respond to a power outage and reroute it to customers in matter of seconds. This action depicts the "selfhealing" aspect of a Smart Grid. The system also isolates the faults in specific pieces of equipment and route power through a backup System in order to maintain power reliability [3].

\section{NEED FOR EFFICIENT ENERGY MANAGEMENT}

Energy efficiency refers to using less energy to provide same or better level of service. The current power distribution networks have been designed for unidirectional power flow from large power plants to users and are not suitable for a massive integration of small or medium renewable power generation plants. The main problems faced by utility companies to sustain efficient energy management are reduction of carbon emissions, increasing energy available for exporting, lowering household energy bills, quick and stable response to power faults occurring in the power grids, a two way communication with the consumers so as to give consumers information about their consumption [4] and how to efficiently utilize the energy and many more. With the introduction of smart grids many of these problems are 
attended to. Smart grids were developed to address the issue of lack of efficient energy utilization by the power grids and to develop a two way communication. Smart grids are a way to get massive amounts of renewable energy with minimal greenhouse emissions. It combines energy from many smaller energy generators like wind turbines, solar panel, micro-turbines, fuel cells and many more to balance out the load of the consumers. Thus the consumers will be able to adjust their power needs according to the variable power generated by the renewables.

\section{REVIEW OF EXISTING SYSTEMS}

The traditional Power Distribution System (PDS) restoration strategies involved a field agent to go to the location where the fault occurred and restore the system manually. The most popular technique used now a days for power restoration is MAS which comprises of various types of agents such as zone agents (ZAs), feeder agents (FAs) and substation agents [5]. MAS uses Q-Learning whose main aim is to look for the best possible solution which will restore as many loads as possible in future by interacting with the dynamic environment and observing its state and then taking the appropriate decision [6]. The hybrid MAS works in a decentralized manner and the agents gain access to information from their immediate neighboring agents. This reduces the dependency of the agents as they are not totally relying on the centralized data. The hybrid MAS works by locating the fault and isolating it using the zone agents and sending the fault to the feeder agents which have the information about their zone agents [7]. The MAS consist of a number of agents interacting with each other using the Foundation for Intelligent Physical Agents (FIPA) standardized Agent Communication Language (ACL). The MAS is used in the Distribution Automation System is a multifunction system which is used to monitor and control the scattered Feeder Remote Terminal Unit (FRTU) in a wide area [8].

The various issues faced during restoration process in the current Power Distribution System are restoration duration estimation, reactive power balance, load generation balance, load and generation co-ordination, monitor and control, protective system, energy storage and system restoration plan [9]. The restoration of smart power grid distribution system can be done with the help of two-way communication (ICT). It is possible to manage the transfer of power at a global level by integrating distributed systems such as MAS using two way communication [10]. A comparison between wired and wireless communication technologies in terms of their data rates and coverage ranges and also on specific network requirements is done which determines methods to incorporate two-way information flow, communication architecture, as well as smart sensing and metering technologies into the current power grid [11].

The existing power system faces many hurdles in terms of power restoration and reconfiguration. Various methods like introducing new IED's, smart meters, enabling demand response, controlling the supply along with further advancements in AI techniques will help make power restoration and reconfiguration more efficient[12]. Distributed multi-agent system approach to solve power restoration problem [13]. Intelligent Optimization Restoration Algorithm with "Face protection mode" is used to identify local topology of fault recovery area and contact switch to reduce search space and amount of computations to complete fault location identification, isolation and restoration quickly [14].New Hierarchically Coordinated
Protection (HCP) approach to mitigate the effects of increased grid complexity. In Predictive protection, statistical data and information from weather related tracking systems, history of the component outages and operating conditions are used to predict fault condition. In adaptive protection, based on feature patterns of waveform measurements obtained online and during learning tripping logic is adjusted. In corrective protection, if local protection is not working properly, accurate and fast fault analysis restores the system components quickly [15].

Thus, the shortcomings of the traditional power systems can be overcome by the use of advanced technologies and algorithms.

\section{INFERENCES DRAWN}

In the existing systems, the restoration time can be reduced by making the restoring process faster and more accurate. The feeder agents can be developed with better learning and decision making capabilities. It must be taken care that the excessive power generation must be more than the loads to be restored.[6] Better and more efficient learning procedures like the adaptive learning can be used in the restoration management.[7] A prioritization strategy can be developed for restoration of loads during power failure. Alternatively, an interaction model can also be developed for the various agents which helps in better negotiation amongst themselves. Measures should be taken to make the Multi-agent System (MAS) more cost efficient.[8] Newer algorithms that take newer topologies like mesh topology into consideration should be developed, unlike the older ones which consider only the tree topology. In large systems, stability issues are more complex while combining the islands. Hence more work needs to be done in this domain. The algorithm for the island synchronism is more complex than the present algorithm. Hence efforts should be taken to simplify it or suggest an alternative. The complexity of solar and power voltage generation and its impact at the time of restoration can also be studied in the future [9]. Ensuring that more than one node can act as a leader to accelerate the system convergence rate. The reliability of the algorithms used can be improved by making sure that the leaders can be easily replaced [11]. For a practical smart grid rollout, a hybrid communication network can be preferred over a centralized or distributed network. The power line communication technologies yield a significant benefit in terms of a minimum cost of network infrastructure investment at the expense of excessive noise and interference coming from the power lines.[16] Hence in order to select the proper technology for the smart grid, various parameters like data rate, security, bandwidth etc. should be considered.[17] This new MAS architecture should be implemented in real life applications and should be tested in more complex scenarios.[18] A more optimized algorithm should be developed while restoring power from traditional restoration system in special cases.[19] Enough power should be provided to satisfy all power demands for all local power committees by considering priorities of local power demands. All this should be done while keeping the cost to its minimum and maintaining the topological and operational constraints. The local power committee can be reorganized into a more hierarchical and flexible architecture. Some approximate heuristics can be applied in the distributed problem solving architecture in which time-critical factor will not allow full communication of the global information. Using measurement information of multiple sites, the control performance can be improved. More focus should be given 
to the operation issues in modern grid like anti-islanding, power quality, short circuit level, reverse power flow and voltage profile. Problem of instability due to sudden increase in the load should be solved. Problems associated with the utilization of distributed generation (DG) like sympathetic trips, unsuccessful solution of faults and unintentional islanding are some other problems faced. For an effective power distribution system, power should be supplied to the right load at the right time. Individual loads and probability of utilization of power can be controlled in supplying critical and non-critical loads [20]. Wireless technologies can be used instead of wired technologies as they provide lower installation cost, more rapid deployment, higher mobility and flexibility [21].

\section{PROPOSED METHODOLOGY}

Looking at various scenario work needs to be done on using fastest restoration techniques. Efficient restoration scheme plays an important role in emergency operation and quick restoration.

Multi Agent System (MAS) framework with capability of learning, for restoration of power uses Q-Learning along with restoration algorithm. It is important to take the advantage of the restoration experience in order make more precise decisions. Q-learning is one of the reinforcement technique. Reinforcement learning agent learns by interacting with its environment. The selection of actions is based on its past experiences and by exploring new choices which can be considered as trial and error learning [6].

Grouping of cases having similar characteristics into clusters Clustering algorithm uses iterative techniques. For creating predictions, exploring data and identifying anomalies in the data these groupings can be used. After identifying relationships a series of clusters are generated based on those relationships. Useful way to visually represent the grouping of data is to use a scatter plot which represents all the cases in the dataset.

Decision Trees algorithm used in predictive modelling of both discrete and continuous attributes. It makes predictions based on relationship between input columns. For the prediction of state of the column it is important to identify the input columns which are correlated with the predictable. Distributed control algorithms can be used in distributed controllers to achieve allocation of electrical power among connected buses.

The control center controls the load by remote switching of individual loads and manages power distribution. Basic advantage for load shedding on smart grid platform is that each load can be independently controlled based on the data available. In intelligent load shedding strategy, prioritization of domestic and commercial loads is considered to improve sustainability and availability of power supply. Optimization of Load shedding using genetic algorithm in smart grid takes revenue loss and social factors into consideration. Grading tool is used to differentiate loads based on importance of load and economic aspect. The genetic algorithm minimizes the load shedding error as well as the impact of the load shedding. Since, the grade points change based on the time of the day, relative importance given to each load also changes at different time of the day. Even if the load shedding requirement is same, different loads are shed at different time of the day [20].

For the successful implementation, appropriate wireless technologies can be Wireless LAN, WiMAX, cellular and spread spectrum radio. Many advanced wireless technologies supporting smart grids are present today. ZigBee is the wireless technology used to provide the unique needs of low-cost sensors as well as control networks. About 40 million homes has now devices connecting to smart meters using ZigBee Smart Energy Protocol [16]. Use of ICT technologies in smart grids can help make the restoration process much more efficient.

Thus the model should focus on protecting the grid by providing quick and efficient restoration solutions through an application of ICT. The proposed model can be aimed towards leveraging an efficient energy management system.

\section{PROPOSED IMPLEMENTATION}

The level 0 or context diagram gives an overview of the system and depicts the flow of the system. It contains various modules such as System, Utilities and Central authority. The transaction of data such as Fault Identification, Power usage, Feedback Etc. from the modules to the central function-"Restoration System" is also shown.

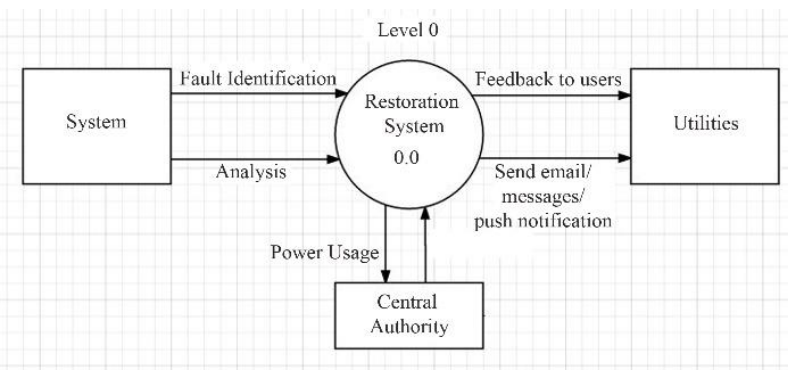

Fig 1: DFD Level 0

The context-level DFD is further expanded into level-1 DFD which shows the working of each module in detail. The "Restoration System" is divided into sub-systems such as Fault Identification, Analysis and Communication. The various data storages used are Records of past solutions, Power generation records, Power utilization records and User database.

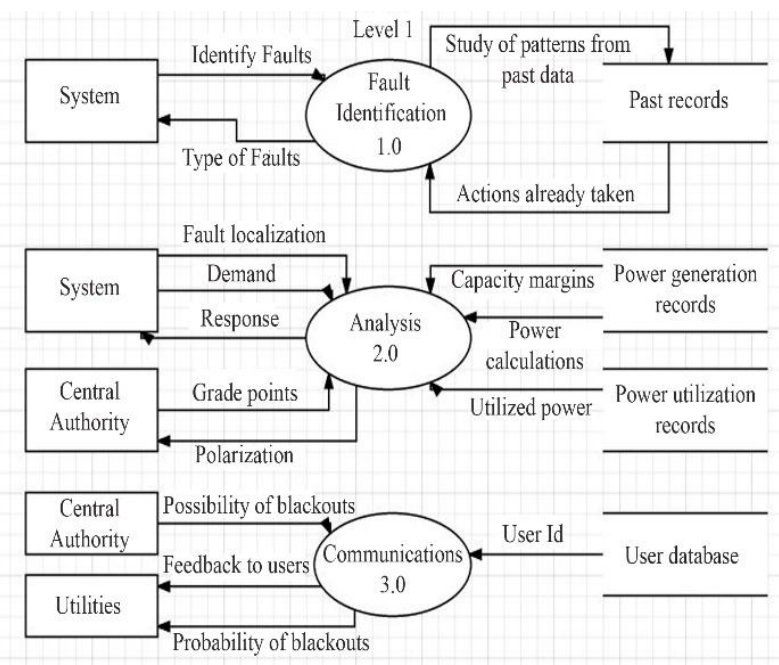

Fig 2: DFD level 1

In level-2 DFD, the Fault Identification module is divided into sub-systems such as Log management, Structure of network, Fast restoration, Load management and Analysis. It gives a more detailed flow of data from the data storages to the various sub-systems and depict all the functionalities related to the module-Fault Identification. 


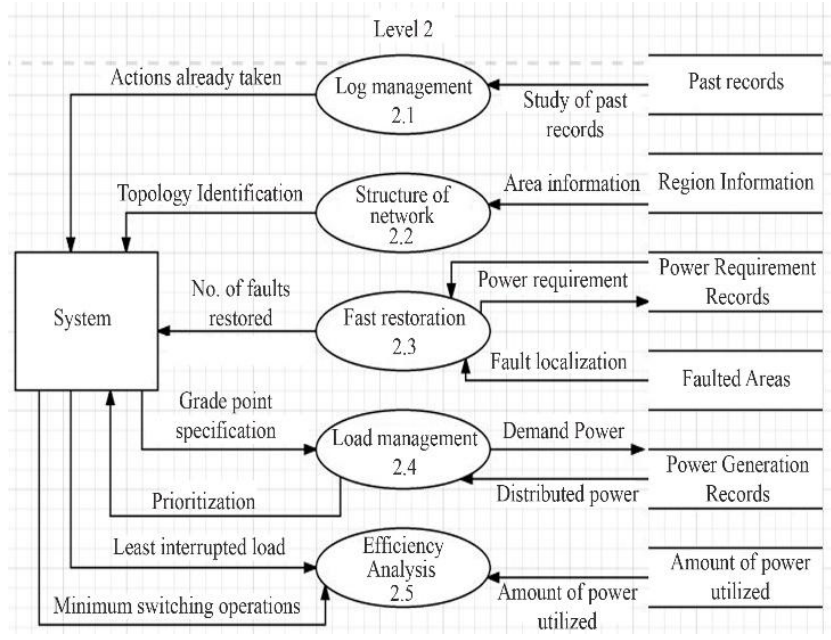

Fig 3: DFD Level 2

\section{CONCLUSION}

After identifying the limitations of existing power grid implementations by survey of literature available, we conclude that technology can be used in power management field for the betterment of society. In this survey paper, we have focused on the inefficient restoration system used in traditional electrical grids and how new and improved restoration techniques used in smart grids provide a quick and efficient restoration. More focus should be given on power transmission and protecting transmission through better restoration techniques.

The next step of this work is to create a network of nodes and switches, identify the fault in the network and implement restoration algorithms that can restore maximum loads efficiently.

\section{REFERENCES}

[1] http://pserc.wisc.edu/research/futuregrid.aspx

[2] http://www.cse.wustl.edu/ jain/cse57410/ftp/grid2/inde x.html

[3] https://www.smartgrid.gov/the_smart_grid/smart_grid.h tml

[4] http://www.energylens.com/articles/energymanagement

[5] Mohammad Jawad Ghorbani et al,"A Multi agent Design for Power DistributionSystems Automation",IEEE proceeding-2015.

[6] Jawad Ghorbani et al,"A MAS Learning Framework for Power Distribution System Restoration", IEEE proceeding 2014.
[7] Sridhar Chouhan et al,"Hybrid Multi Agent approach for Power Distribution System Restoration", IEEE proceedings 2013.

[8] Anisha K et al,"Application of Multi Agent System for Distribution System Automation",IEEE proceeding2014.

[9] Rajan Kumar Mishra et al, "Power System Restoration in Smart Grid Environment”, IEEE proceedings-2014.

[10] R. Belkacemi et al, "Restoration of Smart Grid Distribution System using Two-Way Communication Capability", IEEE proceedings-2013.

[11] Ziang Zhang et al, "Decentralizing the Economic Dispatch Problem using a Two-Level Incremental Cost Consensus Algorithm in a Smart Grid Environment", IEEE proceedings-2011.

[12] Youssef Oualmakran et al, "Opportunities and challenges for smart power restoration and reconfiguration”, IEEE proceedings-2011.

[13] Wan-Yu Yu et al,"Co-ordinating a Society of Switch Agents for Power Distribution Service Restoration in a Smart Grid",IEEE proceeding-2011.

[14] Jianwen Du et al," Power Supply Restoration Algorithm of Multi-Contact Distribution Network with Distributed Intelligent Feeder Automation System”, IEEE proceeding-2014.

[15] Biljana Matic-Cuka et al," Improving Smart Grid Operation with NewHierarchically Coordinated Protection Approach", IEEE proceeding-2012.

[16] Chaiyod Pirak et al,"Recent Advances in Communication Technologies for Smart Grid Application: A Review",IEEE proceeding 2014.

[17] Afshan Mulla et al,"The Wireless Technologies for Smart Grid Communication: A Review",IEEE proceeding 2015.

[18] Mohammad Jawad Ghorbani et al,"A Multi agent Design for Power DistributionSystems Automation", IEEE proceeding-2015.

[19] Z. Song et al,'Restoration of a Smart Distribution System",IEEE proceeding-2010.

[20] K. Uma Rao et al, "A Novel Grading Scheme for Loads to Optimize Load Shedding Using Genetic Algorithm in a Smart Grid Environment”, IEEE proceeding- 2013.

[21] Murat Kuzlu et al, "Assessment of Communication Technologies and Network Requirements for Different Smart Grid Applications", IEEE proceedings-2013. 